\title{
Prognostic potential of IncRNA RHPN1-AS1 in glioma
}

\author{
YONG WU ${ }^{1}$, WUTING WEI ${ }^{2}$, LINJUN TANG $^{1}$ and LIANGWEI WANG ${ }^{1}$ \\ ${ }^{1}$ Department of Neurosurgery, The Second People's Hospital of Wuhu, Wuhu, Anhui 241000; \\ ${ }^{2}$ Department of Neurosurgery, Jinling Hospital, Nanjing Medical University, \\ Nanjing, Jiangsu 210002, P.R. China
}

Received February 11, 2020; Accepted June 11, 2020

DOI: 10.3892/ol.2020.11773

\begin{abstract}
Expression level of long non-coding RNA (lncRNA) RHPN1-AS1 in glioma tissues was detected to determine potential risk factors influencing prognosis of glioma. This study aimed to clarify the molecular mechanisms underlying tumorigenesis of glioma and thus to improve therapeutic efficacy of glioma. RHPN1-AS1 levels in glioma tissues $(n=105)$ and normal brain tissues $(n=105)$ were detected by quantitative real-time polymerase chain reaction (qRT-PCR). The relationship between RHPN1-AS1 level and pathological indicators of glioma patients was analyzed. Glioma patients were followed up for 5 years. Overall survival (OS) and relapse-free survival (RFS) in glioma patients were tested by Kaplan-Meier and log-rank method. Potential factors influencing prognosis of glioma were analyzed by Cox regression model. RHPN1-AS1 was upregulated in glioma tissues. Its level was correlated to histological grade, Karnofsky (KPS) score and postoperative recurrence of glioma patients, rather than sex, age, pathological and tumor size. Glioma patients expressing high level of RHPN1-AS1 suffered worse OS and RFS than those with low level. Advanced histological grade, KPS score $<80$ and high level of RHPN1-AS1 were considered to be risk factors influencing postoperative prognosis of glioma. High level of RHPN1-AS1 is an independent risk factor for poor prognosis of glioma, which may be utilized as a prognostic hallmark.
\end{abstract}

\section{Introduction}

Glioma covers about $40-50 \%$ of primary brain tumors and it is the most common type of malignant tumor in the central nervous system (1). Glioma is fatal because of its diversity, strong invasiveness and heterogeneity (2). Despite the rapid development of medical instruments and surgical techniques in the past decades, the mortality and disability of glioma are still ranked high. Most of glioma patients can only survive

Correspondence to: Dr Yong Wu, Department of Neurosurgery, The Second People's Hospital of Wuhu, 259 Jiuhuashan, Wuhu, Anhui 241000, P.R. China

E-mail: ma7t7u@163.com

Key words: glioma, RHPN1-AS1, prognosis
1-2 years after diagnosis. It is estimated that the progression-free survival of glioma is 6.2-7.5 months, and the median survival is 14.6-16.7 months. The 2-year and 5-year survival of glioma is 27 and $9.8 \%$, respectively (3). To prolong the survival of glioma patients, therapeutic strategies have been largely improved and their efficacies are still far from satisfactory. Developing novel and effective treatments for glioma has received considerable effort and support.

Long non-coding RNAs (LncRNAs) are long-chain, noncoding RNAs that barely encode proteins. They recognize specific DNAs or RNAs to further affect their translation or transcription. Moreover, lncRNAs fold into secondary or higher structures and bind to proteins, thereafter exerting a regulatory role by altering the activities or intracellular localization of corresponding proteins $(4,5)$. It is reported that lncRNA level is closely linked to tumor phenotypes (5). LncRNA has shown potential to be a tumor hallmark. Differentially expressed lncRNAs have been identified in glioma tissues (6). In addition, some lncRNAs are responsible for tumor cell growth and metastasis of glioma (7).

LncRNA RHPN1-AS1 is a transcription product with 2,030 nucleotides (8). It is highly expressed in invasive tumors, including non-small cell lung cancer (NSCLC) and uveal melanoma $(9,10)$. Overexpression of RHPN1-AS1 predicts a poor prognosis of tumor diseases. On the contrary, silence of RHPN1-AS1 suppresses proliferative and metastatic abilities of tumor cells (11). It is reported that IncRNA RHPN1-AS1 drives proliferation, migration and invasion in cervical cancer cells through activating the miR-299-3p/FGF2 axis (12). LncRNA RHPN1-AS1 also triggers the malignant progression of liver cancer (13). In glioma specimens and cell lines, RHPN1-AS1 is highly expressed. Knockdown of RHPN1-AS1 in glioma cells attenuates proliferative, migratory and invasive potentials (14). So far, the potential influence of RHPN1-AS1 on clinical features and prognosis of glioma is rarely reported. In this study, we assessed the prognostic potential of RHPN1-AS1 in glioma patients. Our findings not only provided new directions for clinical diagnosis and monitoring of glioma, but also opened a new field of gene therapy.

\section{Patients and methods}

Patients. A total of 105 cases of glioma tissues were surgically resected from glioma patients undergoing surgery in The Second People's Hospital of Wuhu (Wuhu, China) from June 
A

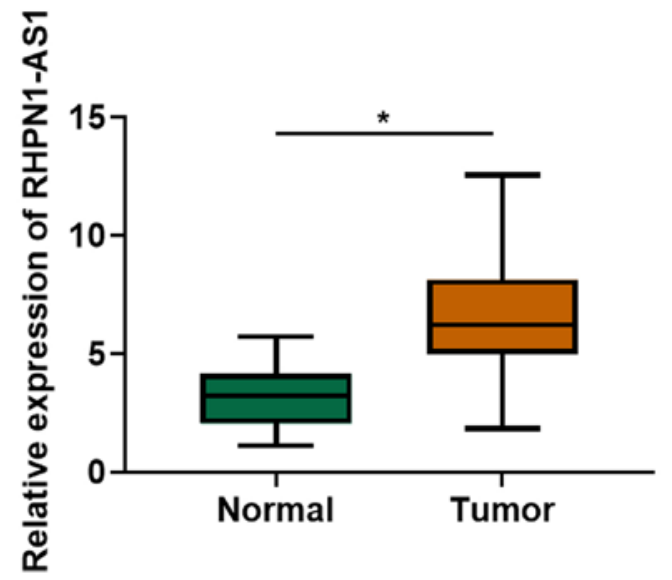

B

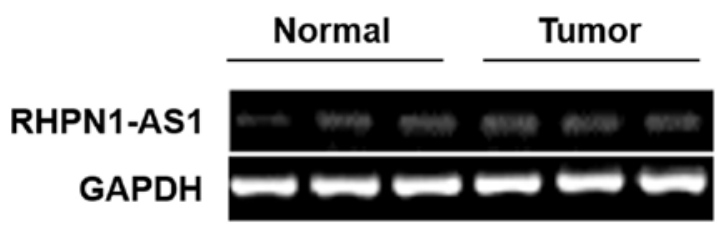

Figure 1. Upregulation of RHPN1-AS1 in glioma. (A) RHPN1-AS1 levels in glioma tissues ( $\mathrm{n}=105)$ and normal brain tissues ( $\mathrm{n}=105$ ) were detected by qRT-qPCR ("P<0.05). (B) The RNA levels of RHPN1-AS1, and GAPDH in three patients and normal brain tissues were determined by RT-PCR. qRT-PCR, quantitative real-time polymerase chain reaction; GAPDH, glyceraldehyde-3-phosphate dehydrogenase.

2017 to December 2019. Among the 105 glioma patients, 59 were males and 46 were females, with a median age of 49.7 years and none of the patients included in the study were epileptic. According to the histological grade classification defined by the criteria of the 10th edition of International Classification of Diseases (ICD-10) (15), 54 cases were grade I-II and 51 were grade III-IV. Furthermore, 105 cases of normal brain tissues were collected from patients undergoing intracranial decompression for trauma in the same period. Subjects with medical history of anti-tumor therapy were excluded. Tissue samples were immediately frozen in liquid nitrogen and preserved at $-80^{\circ} \mathrm{C}$. Patients and their families in this study were fully informed.

The study was approved by the Ethics Committee of The Second People's Hospital of Wuhu. Signed informed consent was obtained from the patients and/or guardians.

$R N A$ preparation and reverse-transcriptase polymerase chain reaction ( $R T-P C R)$. Total RNA was extracted using TRIzol and then treated with RNase R (Epicentre Technologies Corp.). The mixture was incubated at $37^{\circ} \mathrm{C}$ for $1 \mathrm{~h}$. Total RNA $(1 \mu \mathrm{g})$ was transcribed into cDNA by Primescript RT Reagent (Takara Bio, Inc.). The PCR reactions were evaluated with $2 \%$ w/v agarose gels.

Quantitative real-time polymerase chain reaction ( $q R T-P C R)$. Tissues were lysed for isolating total RNA using TRIzol method (Invitrogen; Thermo Fisher Scientific, Inc.). RNA was reversely transcribed into complementary deoxyribose nucleic acid(cDNA) using Primescript RTReagent (TakaraBio,Inc.) and applied for qRT-PCR. Glyceraldehyde 3-phosphate dehydrogenase (GAPDH) was used as the internal reference. RHPN1-AS1, forward: 5'-GCTCCTGGTCATCAAGTTCCTCT-3', reverse: 5'-GCACAGGCACCAGAATGATCC-3'; GAPDH, forward: 5'-TGTTGCCATCAATGACCCCTT-3', reverse: 5'-CTCCAC GACGTACTCAGCG-3'.

Follow up. All subjects were followed up for 5 years through outpatient visit or by telephone. The duration from the date of initial surgery to the death was recorded. OS and RFS were finally calculated. Subjects who died of accidents or causes unrelated to glioma were excluded.

Statistical analysis. Statistical Product and Service Solutions (SPSS) 20.0 (IBM Corp.) was used for data analyses. Data were expressed as mean \pm standard deviation. Differences between two groups were analyzed by the unpaired t-test. Kaplan-Meier method was introduced for survival analysis, followed by log-rank test for comparison. The correlation between RHPN1-AS1 level and pathological indicators of glioma patients was analyzed by Chi-square test. Potential factors influencing prognosis of glioma were analyzed by Cox regression model. $\mathrm{P}<0.05$ was considered as statistically significant.

\section{Results}

Upregulation of RHPN1-AS1 in glioma. RHPN1-AS1 levels in glioma tissues $(n=105)$ and normal brain tissues $(n=105)$ were detected by qRT-PCR. The data showed higher abundance of RHPN1-AS1 in glioma tissues compared with those of normal brain tissues (Fig. 1A and B). It is indicated that RHPN1-AS1 may be involved in the progression of glioma.

Correlation between RHPN1-AS1 level and pathological indicators of glioma patients. According to the cut-off value of RHPN1-AS1 in the collected glioma tissues, the enrolled glioma patients were divided into high-level $(n=49)$ and low-level $(n=56)$ group. By analyzing their pathological data, it was demonstrated that RHPN1-AS1 level was correlated to histological grade (grade III-IV, P<0.001), KPS score (KPS $<80, \mathrm{P}=0.026)$ and postoperative recurrence $(\mathrm{P}=0.010)$ of glioma patients, rather than sex $(\mathrm{P}=0.563)$, age $(\mathrm{P}=0.328)$, pathological $(\mathrm{P}=0.537)$ or tumor size $(\mathrm{P}=0.052)$ (Table $\mathrm{I})$.

Prognostic potential of RHPN1-AS1 in glioma. To further investigate the prognostic potential of RHPN1-AS1 in glioma, Kaplan-Meier method was introduced for assessing OS and RFS 
Table I. Correlation between RHPN1-AS1 level and pathological indicators of glioma patients.

\begin{tabular}{|c|c|c|c|c|c|}
\hline \multirow[b]{2}{*}{ Variables } & \multirow[b]{2}{*}{ No. } & \multicolumn{2}{|c|}{ RHPN1-AS1 } & \multirow[b]{2}{*}{$\chi^{2}$} & \multirow[b]{2}{*}{ P-value } \\
\hline & & $\begin{array}{l}\text { High level } \\
\quad(n=49)\end{array}$ & $\begin{array}{l}\text { Low level } \\
\quad(n=56)\end{array}$ & & \\
\hline \multicolumn{6}{|l|}{ Sex } \\
\hline Male & 59 & 29 & 30 & 0.334 & 0.563 \\
\hline Female & 46 & 20 & 26 & & \\
\hline \multicolumn{6}{|l|}{ Age, years } \\
\hline$<50$ & 44 & 23 & 21 & 0.956 & 0.328 \\
\hline$\geq 50$ & 61 & 26 & 35 & & \\
\hline \multicolumn{6}{|l|}{ Histological grade } \\
\hline I-II & 54 & 15 & 39 & 15.938 & $<0.001$ \\
\hline III-IV & 51 & 34 & 17 & & \\
\hline \multicolumn{6}{|l|}{ Pathological type } \\
\hline Astrocytoma & 32 & 18 & 14 & 1.243 & 0.537 \\
\hline Oligocytoma & 38 & 18 & 20 & & \\
\hline Ependymal tumor & 35 & 21 & 14 & & \\
\hline \multicolumn{6}{|l|}{ KPS score } \\
\hline$\geq 80$ & 55 & 20 & 35 & 4.926 & 0.026 \\
\hline$<80$ & 50 & 29 & 21 & & \\
\hline \multicolumn{6}{|l|}{ Tumor size, $\mathrm{cm}$} \\
\hline$<4$ & 47 & 17 & 30 & 3.767 & 0.052 \\
\hline$\geq 4$ & 58 & 32 & 26 & & \\
\hline \multicolumn{6}{|c|}{ Postoperative recurrence } \\
\hline No & 61 & 22 & 39 & 6.573 & 0.01 \\
\hline Yes & 44 & 27 & 17 & & \\
\hline
\end{tabular}

KPS, Karnofsky.
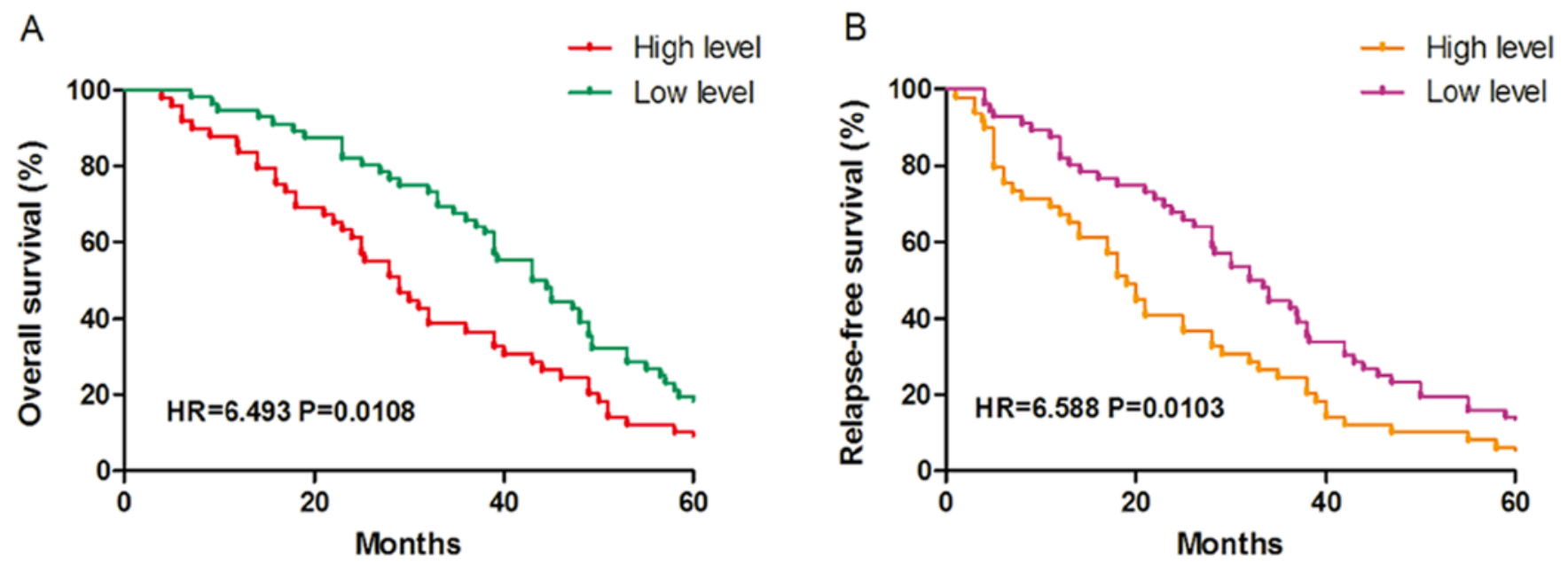

Figure 2. Prognostic potential of RHPN1-AS1 in glioma. (A) Overall survival and (B) relapse-free survival in glioma patients with high or low level of RHPN1-AS1.

in enrolled glioma patients. OS was worse in glioma patients of high-level RHPN1-AS1 group than those in low-level group (HR=6.493, P=0.0108) (Fig. 2A). Similarly, worse RFS was observed in glioma patients of high-level RHPN1-AS1 group as well $(\mathrm{HR}=6.588, \mathrm{P}=0.0103)$ (Fig. 2B). It is suggested that high level of RHPN1-AS1 is unfavorable for prognosis of glioma. 
Table II. COX regression analyses on risk factors influencing the prognosis of glioma.

\begin{tabular}{|c|c|c|c|c|}
\hline \multirow[b]{2}{*}{ Variables } & \multicolumn{2}{|c|}{ Univariate analysis } & \multicolumn{2}{|c|}{ Multivariate analysis } \\
\hline & $\mathrm{HR}(95 \% \mathrm{CI})$ & P-value & $\mathrm{HR}(95 \% \mathrm{CI})$ & P-value \\
\hline Sex & & & & \\
\hline Female/male & $1.014(0.666,1.545)$ & 0.529 & - & - \\
\hline Age & & & & \\
\hline$<50 / \geq 50$ years & $0.815(0.535,1.241)$ & 0.39 & - & - \\
\hline $\begin{array}{l}\text { Histological grade } \\
\text { (I-II/III-IV) }\end{array}$ & $2.173(1.921,3.046)$ & 0.0361 & $1.956(1.276,2.997)$ & 0.0421 \\
\hline Pathological type & & & & \\
\hline Astrocytoma/oligocytoma & $2.114(0.963,5.331)$ & 0.5449 & - & - \\
\hline $\begin{array}{l}\text { KPS score } \\
\geq 80 /<80\end{array}$ & $1.289(1.038,3.931)$ & 0.0287 & $1.217(1.007,3.005)$ & 0.036 \\
\hline $\begin{array}{c}\text { Tumor size } \\
<4 / \geq 4 \mathrm{~cm}\end{array}$ & $1.018(0.670,1.548)$ & 0.933 & - & - \\
\hline $\begin{array}{l}\text { Postoperative recurrence } \\
\text { No/yes }\end{array}$ & $1.347(0.884,2.053)$ & 0.165 & - & - \\
\hline $\begin{array}{l}\text { RHPN1-AS1 } \\
\text { Low/high }\end{array}$ & $1.700(1.119,2.582)$ & 0.013 & $1.589(1.165,2.616)$ & 0.0389 \\
\hline
\end{tabular}

Risk factors of glioma prognosis. To determine the risk factors influencing the prognosis of glioma, univariate and multivariate COX regression analyses were conducted based on the pathological indicators of glioma patients and their 5-year survival. Advanced histological grade, KPS score $<80$ and high level of RHPN1-AS1 were considered to be risk factors influencing postoperative prognosis of glioma (Table II).

\section{Discussion}

Glioma is an intracranial neoplastic disease which is caused by the malignant proliferation of glial cells due to genetic and environmental changes. Clinical manifestations of glioma include headache, vomiting, epilepsy, lalopathy, hemiplegia and paresthesia (16). Severe glioma is fatal because of the occurrence of cerebral hernia. Therefore, it is necessary and urgent to develop effective treatment strategies for glioma (17).

LncRNAs have been verified to be functional mediators (18). Increasing evidence has shown the involvement of abnormally expressed lncRNAs in tumorigenesis and tumor progression (19-21). They extensively participate in the regulation of chromatin modification, transcription or post-transcription mediation $(22,23)$. So far, lncRNAs are considered as a new type of promising hallmarks and therapeutic targets (24). Through microarray screening, lncRNA DANCR is verified to promote the malignant progression of glioma (25). LncRNA ZEB1-AS1 knockdown is able to block glioma cells to proliferate and metastasize (26).
LncRNA RHPN1-AS1 is a key regulatory in many types of tumors. Lu et al (10) reported that RHPN1-AS1 is upregulated in most of uveal melanoma cell lines. In breast cancer, upregulated RHPN1-AS1 is closely associated with advanced staging and poor prognosis (27). In this analysis, RHPN1-AS1 was upregulated in glioma tissues. However, in the present study, the expression of RHPN1-AS1 was detected only by RT-PCR and qRT-PCR, other methods should be used to detect RHPN1-AS1, which was a limitation of our study. Its level was correlated to histological grade, KPS score and postoperative recurrence of glioma patients, rather than sex, age or tumor size. Glioma patients expressing high level of RHPN1-AS1 suffered poor OS and RFS. Cox regression analysis indicated that advanced histological grade, KPS score $<80$ and high level of RHPN1-AS1 were independent risk factors influencing postoperative prognosis of glioma. Additionally, the lack of radiological data is another limitation of the present study. Future studies should be performed to collect radiological data from patients with glioma.

Our findings suggested that RHPN1-AS1 exerts a carcinogenic role in glioma, and it may be used as an independent factor to predict the disease progression of glioma. Nevertheless, potential bias caused by subject collection cannot be ruled out. Larger number of samples to further validate our findings and the molecular mechanism of RHPN1-AS1 in regulating the process of glioma is still required.

In conclusion, high level of RHPN1-AS1 is an independent risk factor for poor prognosis of glioma, which may be utilized as a prognostic hallmark. 


\section{Acknowledgements}

Not applicable.

\section{Funding}

No funding was received.

\section{Availability of data and materials}

All data generated or analyzed during this study are included in this published article.

\section{Authors' contributions}

YW and WW designed the study and performed the experiments. WW and LT acquired the data. LT and LW analyzed the data. YW prepared the manuscript. All authors read and approved the final manuscript.

\section{Ethics approval and consent to participate}

The study was approved by the Ethics Committee of The Second People's Hospital of Wuhu (Anhui, China). Signed informed consent was obtained from the patients and/or guardians.

\section{Patient consent for publication}

Not applicable.

\section{Competing interests}

The authors declare that they have no competing interests.

\section{References}

1. Sathornsumetee S and Rich JN: New treatment strategies for malignant gliomas. Expert Rev Anticancer Ther 6: 1087-1104, 2006.

2. Liu L, Zhang Y, Zhu K, Song L, Tao M, Huang P and Pan Y: Resveratrol inhibits glioma cell growth via targeting LRIG1. J BUON 23: 403-409, 2018.

3. Wang $\mathrm{Y}$ and Lan Q: Long non-coding RNA AFAP1-AS1 accelerates invasion and predicts poor prognosis of glioma. Eur Rev Med Pharmacol Sci 22: 5223-5229, 2018.

4. Batista PJ and Chang HY: Long noncoding RNAs: Cellular address codes in development and disease. Cell 152: 1298-1307, 2013.

5. Reid IR: Short-term and long-term effects of osteoporosis therapies. Nat Rev Endocrinol 11: 418-428, 2015.

6. Han L, Zhang K, Shi Z, Zhang J, Zhu J, Zhu S, Zhang A, Jia Z, Wang G, Yu S, et al: LncRNA profile of glioblastoma reveals the potential role of lncRNAs in contributing to glioblastoma pathogenesis. Int J Oncol 40: 2004-2012, 2012.

7. Maher EA, Furnari FB, Bachoo RM, Rowitch DH, Louis DN, Cavenee WK and DePinho RA: Malignant glioma: Genetics and biology of a grave matter. Genes Dev 15: 1311-1333, 2001.

8. Mo XB, Wu LF, Lu X, Zhu XW, Xia W, Wang L, He P, Bing PF, Zhang YH, Deng FY, et al: Detection of lncRNA-mRNA interaction modules by integrating eQTL with weighted gene co-expression network analysis. Funct Integr Genomics 19 $217-225,2019$
9. Li X, Zhang X, Yang C, Cui S, Shen Q and Xu S: The lncRNA RHPN1-AS1 downregulation promotes gefitinib resistance by targeting miR-299-3p/TNFSF12 pathway in NSCLC. Cell Cycle 17: 1772-1783, 2018.

10. Lu L, Yu X, Zhang L, Ding X, Pan H, Wen X, Xu S, Xing Y, Fan J, Ge S, et al: The long non-coding RNA RHPN1-AS1 promotes uveal melanoma progression. Int J Mol Sci 18: 226, 2017.

11. Xu C, Ping Y, Zhao H, Ning S, Xia P, Wang W, Wan L, Li J, Zhang L, Yu L, Xiao Y. LncNetP, a systematical lncRNA prioritization approach based on ceRNA and disease phenotype association assumptions. Oncotarget 8: 114603-114612, 2017.

12. Duan H, Li X, Chen Y, Wang Y and Li Z: LncRNA RHPN1-AS1 promoted cell proliferation, invasion and migration in cervical cancer via the modulation of miR-299-3p/FGF2 axis. Life Sci 239: 116856, 2019.

13. Fen H, Hongmin Z, Wei W, Chao Y, Yang Y, Bei L and Zhihua S: RHPN1-AS1 drives the progression of hepatocellular carcinoma via regulating miR-596/IGF2BP2 axis. Curr Pharm Des 25: 4630-4640, 2020.

14. Cui P, Su J, Li Q, Xu G and Zhu N: LncRNA RHPN1-AS1 targeting miR-625/REG3A promotes cell proliferation and invasion of glioma cells. OncoTargets Ther 12: 7911-7921, 2019.

15. Wesseling P and Capper D: WHO 2016 Classification of gliomas. Neuropathol Appl Neurobiol 44: 139-150, 2018.

16. Dimberg A: The glioblastoma vasculature as a target for cancer therapy. Biochem Soc Trans 42: 1647-1652, 2014.

17. Wapinski $\mathrm{O}$ and Chang HY: Long noncoding RNAs and human disease. Trends Cell Biol 21: 354-361, 2011

18. Yang Z, Lu Y, Xu Q, Tang B, Park CK and Chen X: HULC and H19 played different roles in overall and disease-free survival from hepatocellular carcinoma after curative hepatectomy: A preliminary analysis from gene expression omnibus. Dis Markers 2015: 191029, 2015.

19. Li J, Meng H, Bai Y and Wang K: Regulation of lncRNA and its role in cancer metastasis. Oncol Res 23: 205-217, 2016.

20. Li C, Lei B, Huang S, Zheng M, Liu Z, Li Z and Deng Y: H19 derived microRNA-675 regulates cell proliferation and migration through CDK6 in glioma. Am J Transl Res 7: 1747-1764, 2015.

21. Wang Y, Wang Y, Li J, Zhang Y, Yin H and Han B: CRNDE, a long-noncoding RNA, promotes glioma cell growth and invasion through mTOR signaling. Cancer Lett 367: 122-128, 2015.

22. Zhang Y, Sun Y, Peng R, Liu H, He W, Zhang L, Peng H and Zhang Z: The long noncoding RNA 150Rik promotes mesangial cell proliferation via miR-451/IGF1R/p38 MAPK signaling in diabetic nephropathy. Cell Physiol Biochem 51: 1410-1428, 2018.

23. Dong X, Fang Z, Yu M, Zhang L, Xiao R, Li X, Pan G and Liu J: Knockdown of long noncoding RNA HOXA-AS2 suppresses chemoresistance of acute myeloid leukemia via the miR-520c-3p/ S100A4 Axis. Cell Physiol Biochem 51: 886-896, 2018.

24. Powers AS: Adult neurogenesis in mammals and nonmammals. Commentary on Kempermann G (2012): New neurons for 'survival of the fittest'. Nat Rev Neurosci 13:727-736. Brain Behav Evol 81: 206-208, 2013.

25. Weng J, Tu M, Wang P, Zhou X, Wang C, Wan X, Zhou Z, Wang L, Zheng X, Li J, et al: Corrigendum to 'Amiodarone induces cell proliferation and myofibroblast differentiation via ERK1/2 and p38 MAPK signaling in fibroblasts' [Biomed. Pharmacother. 115 (2019) 108889]. Biomed Pharmacother 118: 109085, 2019.

26. Wei $\mathrm{N}$, Wei $\mathrm{H}$ and Zhang $\mathrm{H}$ : Long non-coding RNA ZEB1-AS1 promotes glioma cell proliferation, migration and invasion through regulating miR-577. Eur Rev Med Pharmacol Sci 22: 3085-3093, 2018.

27. Zheng S, Lv P, Su J, Miao K, Xu H and Li M: Silencing of the long non-coding RNA RHPN1-AS1 suppresses the epithelial-tomesenchymal transition and inhibits breast cancer progression. Am J Transl Res 11: 3505-3517, 2019.

This work is licensed under a Creative Commons Attribution-NonCommercial-NoDerivatives 4.0 International (CC BY-NC-ND 4.0) License. 